\section{REFERENCES}

Eimas, P. D. Effects of overtraining, irrelevant stimuli and training task on reversal discrimination learning in children. Journal of Experimental Child Psychology, 1966, 3, 315-323.

Hess, R. D., \& Shipman, V. C. Early experience and the socialization of cognitive modes in children. Child Development, 1965, 36, 869-886.

Kagan, J., Moss, H. A., \& Siegel, I. E. Psychological significance of styles of conceptualization. Monograph of Society for Research in Child Development, 1963, 28, 73-112.

Kagan, J., Rosman, B. L., Day, D., Albert, J., \& Phillips, W. Information processing in the child: Significance of analytic and reflective attitudes. Psychological Monographs, 1964, 78, 1-37.

Kendler, T. S. Verbalization and optional reversal shifts among kindergarten children. Journal of Verbal Learning \& Verbal Behavior, 1964, 3, 428-436.

Kendler, T. S. Development of mediating responses in children.
In P. H. Mussen, J. J. Conger, and J. Kagan (Eds.), Readings in child development and personality. New York: Harper \& Row, 1970.

Kendler, H. H., \& Kendler, T. S. Reversal and nonreversal shifts in kind ergarten children. Journal of Experimental Psychology, $1959,58,56-60$.

Kendler, H. H., \& Kendler, T. S. Vertical and horizontal processes in problem solving. Psychological Review, 1962, 69, 1-16.

Lowenkron, B., \& Briessen, E. C. Solution mode in concept identification problems and magnitude of the overlearning reversal effect. Journal of Experimental Psychology, 1971, 89, 85-91.

\title{
Verbal-discrimination learning as a function of encoding variability
}

\author{
JOHN H. MUELLER, EDWARD J. PAVUR, and ROBERT M. YADRICK \\ University of Missouri, Columbia, Missouri 65201
}

\begin{abstract}
The encoding variability of the right (R) and wrong (W) terms was manipulated in a verbal-discrimination task. When the number of different associates defined variability, pairs with low-encoding variability for both $R$ and $W$ terms were learned fastest, followed by pairs with high-encoding variability in both $R$ and $W$ terms. When the number of dictionary meanings defined variability, pairs with high-encoding variability $R$ terms and $W$ terms were learned slower than all other combinations. The outcomes do not correspond to expectations involving only the number of encodings in each component of a pair; it is suggested that the number of nonoverlapping encodings is more important.
\end{abstract}

The frequency theory of verbal-discrimination learning (VDL) assumes that Ss utilize a subjective difference in the frequency of experience for the right $(R)$ and wrong (W) terms in each pair in the list (Ekstrand, Wallace, \& Underwood, 1966). The evidence in support of the basic tenets of the theory is impressive (cf. Wallace, 1972). The present research is concerned with an aspect of VDL not thoroughly considered to this point, namely the encoding variability of the items in the pairs.

Frequency theory assumes that the S's experiences, e.g., perceiving, pronouncing, rehearsing, etc., accumulate as the $S$ attends to either the $\mathrm{R}$ term or $\mathrm{W}$ term in each pair. However, to the extent that the pair units may be perceived or encoded differently on separate occasions, these experiences may not accrue to the same encoding each time. If experiences for each encoding are, in fact, treated independently (rather than additively) in choosing a response, then the expectations of frequency theory would vary somewhat depending upon this aspect of the materials. This basic interpretation follows from a feature analysis of VDL (e.g., Kausler, 1973, 1974), but it also follows from frequency theory if one grants the common distinction between nominal and functional stimuli.

The basic encoding-variability hypothesis suggests several predictions for variation in the number of encodings in $\mathrm{R}$ terms and $\mathrm{W}$ terms. First, $\mathrm{R}$ terms with several possible encodings would have their experiences 
distributed across features perhaps, with the result being that any one encoding would have fewer frequency units attached to it than would be expected on the basis of the number of trials, etc. Increasing R-term encoding variability should thus lead to slower learning, provided W-term encoding variability is held constant. Similarly, $\mathrm{W}$ terms which have multiple encodings would have their experiences distributed across various features, but this should lead to more rapid acquisition, holding R-term encoding variability constant. That is, the difference between the $\mathrm{R}$ term and the experiences to any one encoding of the $\mathrm{W}$ term then becomes greater than would be the case if all W-term experiences were concentrated in one encoding.

Thus, increasing encoding variability should help VDL if it occurs for the $\mathrm{W}$ terms, but hinder for $\mathrm{R}$ terms. Of course, the opposite predictions would follow when encoding variability decreases. In fact, the best VDL performance should result for pairs where the R-term encoding variability is low, concentrating all experiences in one encoding, and where the W-term encoding variability is high, distributing those experiences across encodings. Similar reasoning suggests that the opposite arrangement, low W-term encoding variability and high R-term encoding variability, would lead to the worst VDL performance.

To the extent that high meaningfulness corresponds to low-encoding variability (e.g., Martin, 1968), this should help in VDL when the $\mathrm{R}$ terms are high meaningfulness, but hinder when $\mathrm{W}$ terms are low meaningfulness. Williams and Underwood's (1970) second experiment failed to find meaningfulness effects in frequency judgments, and the VDL literature is mixed. Facilitation generally results when meaningfulness is varied such that one member of a pair is high and the other member is low in meaningfulness throughout the same list, but not for the case where both members of a pair are high meaningfulness compared to other pairs with both members low meaningfulness throughout the whole list (e.g., Ullrich \& Balogh, 1972).

There is evidence that other attributes of the materials produce results that appear to be basically consistent with the preceding analysis. For example, Kausler (1973) found that highly distinctive orthography facilitated VDL, and Schulz and Lovelace (1972) found that high acoustic and formal similarity retarded VDL. Furthermore, while their experiment did not involve VDL directly, Underwood and Zimmerman's (1973) analysis of words into syllables also seems encouraging. They found that frequency indeed appeared to accrue to individual syllables of words, in accord with a basic distinction between the nominal and the functional locus of accumulated experiences.

The present experiments were intended as further tests of the encoding variability hypothesis, using two other indices of encoding variability. Butler and Merikle (1970) have scaled trigrams for the number of different associations elicited, and this served as the index in Experiment 1, controlling for meaningfulness. Likewise, the number of dictionary meanings a word possesses also seems a likely index of alternative encodings, and this was used in Experiment 2. Both of these indices seem intuitively as related to the number of encodings as meaningfulness.

\section{METHOD}

\section{Experiment 1}

Design and Subjects. The basic design was a 3 by 2 by 2 mixed factorial, with instructions (control, associates, features) as a between-Ss factor, and R-term encoding variability (low, high) and W-term encoding variability (low, high) as within-Ss factors, with 14 Ss per cell.

Materials. A 16-pair list was formed from 32 consonant vowel - consonant trigrams chosen from the Butler and Merikle (1970) norms as follows. The two trigrams producing the greatest number of different associations at each level of meaningfulness from .05 to .75 were chosen as the high-encoding variability items, 16 in all. Similarly, the two trigrams at those levels producing the fewest different associations were chosen as the low-encoding variability items. The 16 high- and 16 low-encoding variability items were then formed into pairs. There were four types of pairs formed by the possible combinations of high- and low-encoding variability in the $R$ terms and $\mathrm{W}$ terms, respectively: high-high, high-low, low-high, low-low. There were four pairs of each type, with the average meaningfulness of each type equated (.40). The use of a "mixed" list thus reduces any selection strategy which would be possible if the entire W-term set was distinctive compared to the R-term set.

Procedure. Each S performed by the study-test method for eight trials. The list was arranged in eight different orders, with equal left-right distribution of $\mathrm{R}$ terms within a trial, and each word appearing on the left and right equally over trials. The pairs were presented visually at a 5 -sec rate, with the $R$ term underlined, followed by a 45 -sec recall period, during which Ss circled the $R$ term $s$ in the pairs listed on the sheet of paper.

One-third of the Ss were given initial instructions that thinking of associates to the $\mathrm{R}$ terms would be helpful, and another third were told that thinking of features of the $R$ terms would be helpful. It was thought that these instructions might accentuate the encoding variability differences, but no such effects resulted, so this factor will not be pursued in any detail.

\section{Experiment 2}

Except for the materials used, and the number of Ss (54), this experiment was exactly like Experiment 1 . In this case, the number of dictionary meanings constituted the definition of encoding variability. Fifty-five high-frequency concrete nouns comprised the basic word pool. The number of dictionary meanings was determined for each using the Funk and Wagnall Standard College Dictionary, deleting archaic meanings. The 16 words with the greatest number of dictionary meanings were chosen, and the 16 with the fewest meanings. These were then formed into pair types as before. The instructional manipulation was also included in the design, but again produced no effects.

\section{RESULTS}

\section{Experiment 1}

The number of correct responses were analyzed in a 3 (instructions) by 2 (R-term encoding variability) by 2 (W-term encoding variability) by 8 (trials) layout. The instructions main effect and interactions with 


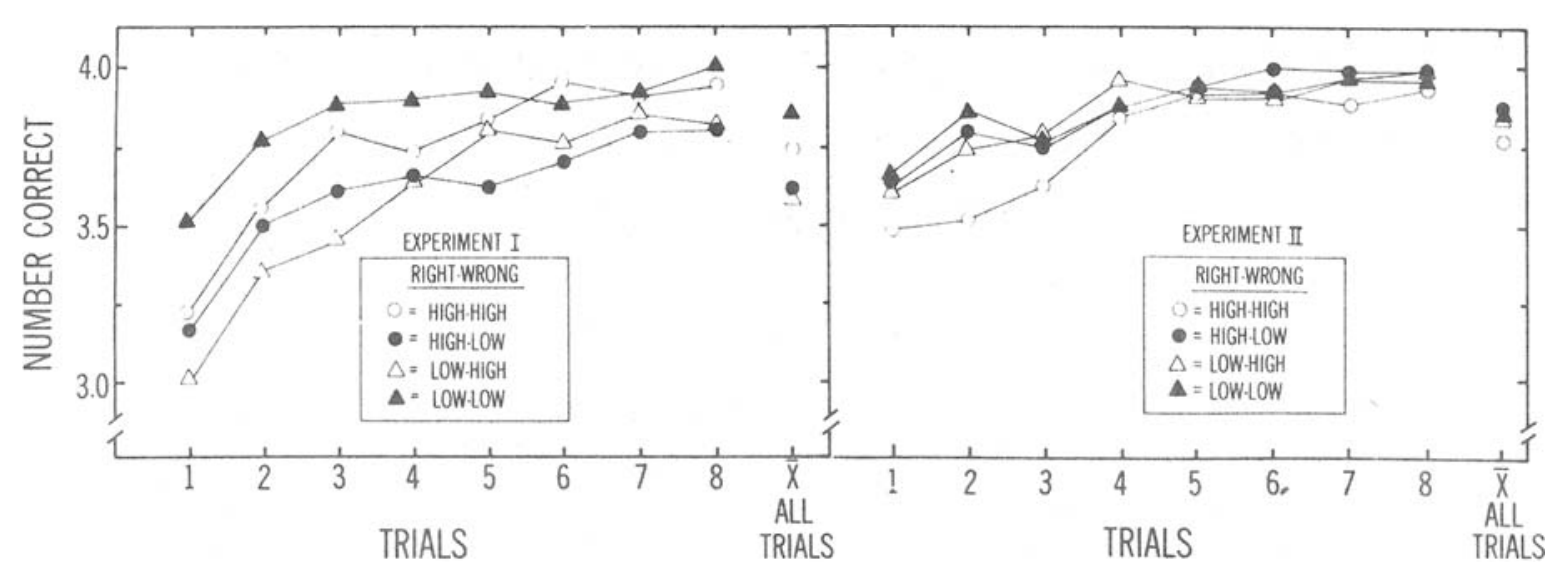

Fig. 1. Average number correct by trials in Experiment 1 (left panel) and Experiment 2 (right panel), by pair type.

instructions produced Fs $<1.78$. While the R-term and W-term main effects failed to reach significance, $F_{s}(1,39)=1.36$ and 2.10 , respectively, their interaction was significant, $\mathrm{F}(1,39)=24.63, \mathrm{p}<.001$.

These results are shown in the left panel of Fig. 1. As may be seen, the low-low pairs were acquired most rapidly, followed by the high-high pairs, with the low-high pairs initially the worst, and the high-low pairs ultimately the last to be learned. In terms of individual comparisons, the low-low pairs were better than the low-high and high-low pairs $(\mathrm{p}<.01)$, but not the high-high pairs, while the high-high pairs did not differ from low-high and high-low pairs.

\section{Experiment 2}

The analysis of the number correct in Experiment 2 revealed no main effects due to R-term or W-term encoding variability, $F s(1,51)=1.90$ and 3.97, respectively, but their interaction was again significant, $\mathrm{F}(1,51)=5.44, \mathrm{p}<.05$. As may be seen in the right panel of Fig. 1, the only significant difference was the general inferiority for the high-high pairs.

\section{DISCUSSION}

The results of these experiments do not appear to support the initial expectations. The low-high pairs did not demonstrate any superiority, nor did the high-low pairs show any inferiority. The high-high pairs were learned more slowly in Experiment 2, but not in Experiment 1. While the differences in results across the experiments may be attributable in part to the differences in materials, e.g., the necessarily higher formal similarity of the trigrams, neither experiment is in clear accord with the expectations derived from differences in the number of encodings. While the item pools used here may be at fault, it seems likely to us that a more fundamental problem exists.

Whereas the basic encoding variability notion emphasizes the number of features, Kausler (1974) has argued that the critical factor in multiple-item recognition is feature overlap between the $\mathrm{R}$ and $\mathrm{W}$ terms in each pair. In other words, the absolu te number of features is less critical than the number of unshared features between $R$ and $W$ terms. Viewed in this way, the expectations stated initially hold only when the R and W terms share no features in common. When features are shared, the same rank ordering would probably be expected for high-high pairs vs low-low pairs, but predictions become problematic for low-high and high-low pair types. In the latter cases, the problem is how many of the features of the low component are shared by the high component, and then whether the shared or unshared features are experienced at the outset. Unfortunately, this overlap could not be identified a priori here.

However, these are some materials, e.g., homonyms (Kausler \& Olson, 1969), where specific shared features can be manipulated, and studies using such materials seem required to adequately test the notion of feature overlap. In addition, Underwood and Zimmerman's syllable analysis seems potentially useful here. Alternatively, norms for the distinctiveness of words when prired, rather than each individually, might be developed. In any event, it seems likely that a feature overlap interpretation is to be preferred over simply counting separate encodings.

\section{REFERENCES}

Butler, B. E., \& Merikle, P. M. Uncertainty and meaningfulness in paired-associate learning. Journal of Verbal Learning \& Verbal Behavior, 1970, 9, 634-641.

Ekstrand, B. R., Wallace, W. P., \& Underwood, B. J. A frequency theory of verbal discrimination learning. Psychological Review, 1966, 73, 556-578.

Kausler, D. H. Orthographic distinctiveness of homonyms and the feature-tagging hypothesis. American Journal of Psychology, 1973, 86, 141-144.

Kausler, D. H. Continuity of processes across variants of recognition learning. In $R$. L. Solso (Ed.), Theories of cognitive psychology: The Loynla symposium. Potomac: Lawrence Erlbaum, 1974.

Kausler, D. H. \& Olson, R. D. Homonyms as items in verbal discrimination learning and transfer. Journal of Experimental Psychology, 1969, 82, 136-142.

Martin, E. Stimulus meaningfulness and paired-associate transfer: An encoding-variability hypothesis. Psychological Review, 1968, 75, 421-441.

Schulz, L. S., \& Lovelace, E. A. Interpair acoustic and formal similarity in verbal discrimination learning. Journal of Experimental Psychology, 1972, 94, 295-299.

Ullrich, J. R., \& Balogh, B. A. Imagery and meaningfulness of right and wrong items in verbal discrimination learning. Psychonomic Science, 1972, 29, 68-70.

Underwood, B. J., \& Zimmerman, J. The syllable as a source of error in multisyllable word recognition. Journal of Verbal Learning \& Verbal Behavior, 1973, 12, 701-706.

Wallace, W. P. Verbal discrimination. In C. P. Duncan, L. Sechrest, and A. W. Melton (Eds.), Human memory: Festschrift for Benton J. Underwood. New York: Appleton-Cen tury-Crofts, 1972.

Williams, R. F., \& Underwood, B. J. Encoding variability: Tests of the Martin hypothesis. Journal of Experimental Psychology, 1970, 86, 317-324.

(Received for publication April 10, 1974.) 